\title{
Chaos in Plankton Models: Foraging Strategy and Seasonal Forcing
}

Roger Cropp, Faculty of Environmental Sciences, Griffith University, Nathan, Queensland, Australia,

Irene M. Moroz, Mathematical Institute, University of Oxford, Andrew Wiles Building, ROQ, Oxford OX2 6GG, UK,

John Norbury, Mathematical Institute, University of Oxford, Andrew Wiles Building, ROQ, Oxford OX2 6GG, UK

April 7, 2016 


\begin{abstract}
The dynamics of plankton ecosystems have long been of interest to ecologists and mathematicians, with some of the earliest examples of chaotic dynamics being provided by ecological models. Mortality terms were initially identified as determinants of chaos in simple ecosystem models, but relatively little attention has been given to the role of grazing terms. The behaviour of omnivores has arisen as a particularly interesting case. Recent experiments have revealed that plankton omnivores may change their feeding behaviour in response to changes in temperature, and is therefore of interest to plankton modellers contributing models of biogeochemical cycling in the ocean to climate models. In this paper we consider the role of an omnivorous zooplankton's foraging strategy, seasonal variations and the choice of functional forms on the dynamics of a simple two prey-one predator plankton model, within a Conservative-Normal framework. We find that assumptions about the way the predator forages for food, the specific form for grazing and mortality terms, and seasonal changes in the environment all qualitatively affect the predictions that the model will produce. In particular, discriminate foraging and seasonal variations engender chaotic dynamics while Holling Type III grazing and quadratic mortality terms suppress chaotic dynamics.
\end{abstract}

KEYWORDS Plankton modelling, seasonal forcing, chaotic dynamics 


\section{Introduction}

Phytoplankton and zooplankton together form the basis of the oceans' food chain. Through photosynthesis, limited by the availability of dissolved nutrients and solar irradiance, phytoplankton convert carbon dioxide from the Earth's atmosphere, drawing it down into the deep oceans, in the process producing half of the world's atmospheric oxygen (Bork et al. [2015]). Phytoplankton are the primary organic food source for zooplankton, which are in turn the food source for fish and whales.

The potential importance of plankton as key indicators of climate change cannot be underestimated, since the viability of various phytoplankton depends upon water temperature and acidity. Long term climate change could affect phytoplankton populations, their seasonal blooms and/or their extinction (Falkowski [2012]) and in turn, feed back on climate change through changes in biogeochemical cycling of climatically important compounds such as carbon dioxide and dimethylsulphide (Charlson et al. [1987]). PlankTOM5 (Le Quéré et al. [2005]) and PlankTOM10 (Kwiatkowski et al. [2014]) are two examples of global marine ecosystem models, containing many different plankton functional types, aimed at studying the interactions between ocean biogeochemistry and climate change (Mitra et al. [2014]). Recent analysis of data has shown the complexity of plankton grazing interactions (Flynn et al. [2013]), and its sensitivity to changes in ocean temperature (Wilken et al. [2013]).

The Conservative-Normal approach involves a set of ecological rules that characterise the food web, and from which properties of the system can be determined. It was first formulated in a series of papers by Cropp and Norbury (Cropp and Norbury [2012a, 2012b]) up to two different phytoplankton and two different zooplankton populations with a single nutrient constraining the populations. They used Holling type 
II functional forms. Bates et al. [2015] used the Conservative-Normal framework to develop a system of twenty-one coupled nonlinear ordinary differential equations to model a near-shore Antarctic food web. As well as various types of phytoplankton, zooplankton and benthic feeders, the model included fish, whales, seals, penguins and seabirds. The choice of Holling Type III functional forms and nonlinear mortality functions, cast in a Kolmogorov framework, ensured the structural coexistence of each member of the ecosystem as every boundary critical point of the system (where one or more populations were extinct) was locally unstable.

Cropp and co-workers (Cropp et al. [2015], Moroz et al. [2015]) have investigated the role of different types of grazer predation in a prey-prey- predator model with two phytoplankton populations (prey) and one zooplankton population (predator) with Holling type II functional forms. Linear stability and bifurcation analyses showed that under indiscriminate predation (where the predator searches for and eats prey indiscriminately according to what it encounters), only steady states or simple periodic limit cycle solutions obtain. For discriminate predation (where the predator preferentially searches for a particular prey, but will eat other prey that it encounters), more complicated periodic as well as chaotic states are also possible.

Here we extend the analysis to consider a transitionalist model, which contains these two limits as special cases. We utilise our foraging transition parameter with the commonly used closure parameter (the mortality coefficient of the zooplankton) to produce a two-parameter bifurcation diagram, summarising the nonlinear dynamics and placing the two special cases into context. We also consider the effects of seasonal forcing on the system under both discriminate and in discriminate grazing and finally, vary the underlying functional forms of the grazing terms from Holling Type II to 
Holling Type III and the mortality terms from linear to nonlinear to extend our investigation to models with structural coexistence.

\section{The Plankton model}

We investigate a model comprising two different phytoplankton populations, $P_{1}, P_{2}$, being grazed upon by one zooplankton population, $Z$, in the presence of a limiting nutrient $N$ (Cropp and Norbury [2009], Cropp et al. [2015], Moroz et al. [2015]):

$$
\begin{aligned}
\dot{P}_{1} & =P_{1}\left[\frac{\mu_{1} N}{N+\kappa_{1}}-\frac{\phi_{1} Z}{1+\epsilon_{1} P_{1}+\rho \epsilon_{2} P_{2}}-\sigma_{1}\right]=P_{1} f\left(P_{1}, P_{2}, Z\right) \\
\dot{P}_{2} & =P_{2}\left[\frac{\mu_{2} N}{N+\kappa_{2}}-\frac{\phi_{2} Z}{1+\rho \epsilon_{1} P_{1}+\epsilon_{2} P_{2}}-\sigma_{2}\right]=P_{2} g\left(P_{1}, P_{2}, Z\right) \\
\dot{Z} & =Z\left[\frac{\phi_{1}\left(1-\psi_{1}\right) P_{1}}{1+\epsilon_{1} P_{1}+\rho \epsilon_{2} P_{2}}+\frac{\phi_{2}\left(1-\psi_{2}\right) P_{2}}{1+\rho \epsilon_{1} P_{1}+\epsilon_{2} P_{2}}-\sigma_{Z}\right]=Z h\left(P_{1}, P_{2}, Z\right),
\end{aligned}
$$

together with the nutrient $N$ mass conservation condition:

$$
\dot{N}=-\dot{P}_{1}-\dot{P}_{2}-\dot{Z}
$$

where $P_{1}+P_{2}+Z+N=1$. Here the populations $P_{i}$ and $Z$ are measured by the fractions of limiting nutrient bound into their biomass $-N$ is then the fraction of limiting nutrient (available to the populations) in the nutrient pool. Apart from $\rho$, the various parameters appearing in (2.1) are explained in Table 1, together with their measured parameter values in the field (see Cropp and Norbury [2009] for further details). When $\rho=0$, we obtain the discriminate model considered by (Cropp and Norbury [2009]), while for $\rho=1$, we obtain the indiscriminate model considered by 
Cropp et al. [2015] and Moroz et al. [2015] We term (2.1) the transitional model for which $0 \leq \rho \leq 1$. The $\rho=0$ and $\rho=1$ basic models have ecological significance, while the transitional model enables us to put the two limits into context: we think of $\rho$ as a bifurcation parameter. We extend our bifurcation analysis to $\rho=1.4$ to capture the complete behaviour of the model.

\begin{tabular}{|c|c|c|}
\hline Par. & Process & Value \\
\hline$\mu_{1}$ & $\begin{array}{l}\text { Maximum (seasonal average) rate } \\
\text { of } N \text { uptake by } P_{1}\end{array}$ & 1.00 \\
\hline$\mu_{2}$ & $\begin{array}{l}\text { Maximum (seasonal average) rate } \\
\text { of } N \text { uptake by } P_{2}\end{array}$ & 1.15 \\
\hline$\kappa_{1}$ & $\begin{array}{l}\text { Half-saturation constant for } N \\
\text { uptake by } P_{2}\end{array}$ & 0.25 \\
\hline$\kappa_{2}$ & $\begin{array}{l}\text { Half-saturation constant for } N \\
\text { uptake by } P_{2}\end{array}$ & 0.07 \\
\hline$\phi_{1}$ & $Z$ grazing rate on $P_{1}$ & 6.18 \\
\hline$\phi_{2}$ & $Z$ grazing rate on $P_{2}$ & 1.85 \\
\hline$\epsilon_{1}$ & $\begin{array}{l}\text { Half-saturation constant for } Z \\
\text { uptake of } P_{1}\end{array}$ & 5.50 \\
\hline$\epsilon_{2}$ & $\begin{array}{l}\text { Half-saturation constant for } N Z \\
\text { uptake of } P_{2}\end{array}$ & 5.50 \\
\hline$\sigma_{1}$ & $P_{1}$ specific mortality rate & 0.00 \\
\hline$\sigma_{2}$ & $P_{2}$ specific mortality rate & 0.26 \\
\hline$\sigma_{Z}$ & $Z$ specific mortality rate & 0.19 \\
\hline$\psi_{1}$ & $\begin{array}{l}\text { Proportion of } P_{1} \text { uptake excreted } \\
\text { by } Z\end{array}$ & 0.40 \\
\hline$\psi_{2}$ & $\begin{array}{l}\text { Proportion of } P_{2} \text { uptake excreted } \\
\text { by } Z\end{array}$ & 0.40 \\
\hline
\end{tabular}

Table 1: The parameters in (2.1), their interpretations and values

The transitional model (2.1) also satisfies the Conservative-Normal (CN) parameter constraints (see Cropp and Norbury [2009], Cropp et al. [2015]). The resource 
conditions that $f>0$ and $g>0$ when $N=1$ and $Z=0$ give

$$
\sigma_{1}<\frac{\mu_{1}}{1+\kappa_{1}}, \quad \sigma_{2}<\frac{\mu_{2}}{1+\kappa_{2}}
$$

which translates to $0<\sigma_{1}<0.8$ and $0<\sigma_{2}<1.07$ for the measured values in Table 1. Requiring $h>0$ at the extremities of $P_{1}+P_{2}=1$ gives

$$
\sigma_{Z}<\min \left\{\frac{\phi_{1}\left(1-\psi_{1}\right)}{1+\epsilon_{1}}, \frac{\phi_{2}\left(1-\psi_{2}\right)}{1+\epsilon_{2}}\right\}
$$

so that $Z$ survives on either $P_{1}$ or $P_{2}$ (or a combination of the two): a 'facultative omnivore'. This is part of a 'normal' ecology. From Table 1 this gives $0<\sigma_{Z}<0.17$. However when

$$
0.17<\sigma_{Z}<\max \left\{\frac{\phi_{1}\left(1-\psi_{1}\right)}{1+\epsilon_{1}}, \frac{\phi_{2}\left(1-\psi_{2}\right)}{1+\epsilon_{2}}\right\}
$$

we obtain an 'exotic' ecology, and $Z$ becomes an 'obligate omnivore' for $0.17<\sigma_{Z}<$ 0.57: $Z$ grazes on both $P_{1}$ and $P_{2}$, but $P_{1}$ must be present for $Z$ to survive, so that $Z$ is obligate on $P_{1}$.

While (2.5) violates the resource condition on the sign of $h$ at $P_{2}=1$, bifurcation and stability analyses in Moroz et al [2015] show that with the Table 1 measured parameter values, the 'min' constraint permits only two-dimensional oscillatory behaviour in the $\left(P_{2}, Z\right)$-plane, whereas the 'max' constraint allows three-dimensional periodic oscillations in $\left(P_{1}, P_{2}, Z\right)$-space, as well as period-doubling bifurcations and chaos.

The foraging strategy parameter $\rho$, that transitions from discriminate to indiscriminate grazing, and the zooplankton mortality parameter $\sigma_{Z}$, that determines the 
prey-dependence of the zooplankton and also closes the model, provide natural bifurcation parameters with which to investigate the dynamical properties of the model. While $\sigma_{Z}$ transitions smoothly and we can identify critical values for it at which the trophic classification of the zooplankton population changes, the foraging parameter $\rho$ is only explicitly ecologically defined for $\rho=0$ and $\rho=1$. However, we will show that the system smoothly transitions between $\rho=0$ and $\rho=1$, and argue that these transition values might reasonably be interpreted to represent transitional foraging strategies. We also extend the examination of the linear stability boundaries of the system for $\rho>1$ for reasons that are detailed in $\S 3.7$ and Figure 1 . These values represent enhanced indiscriminate foraging, where the foraging efficiency exceeds the handling time.

\section{Critical Points}

The linear stability analysis of the critical points of (2.1) and (2.2) uses the same notation and labelling as Cropp and Norbury [2009]. Indeed the analyses for the origin and prey-only critical points are identical.

\subsection{Origin Critical Point}

There is a critical point at the origin, given by $\left(P_{1}, P_{2}, Z, N\right)=(0,0,0,1)$, whose linear stability is determined from the eigenvalues

$$
\lambda_{01}=\frac{\mu_{1}}{1+\kappa_{1}}-\sigma_{1}, \quad \lambda_{02}=\frac{\mu_{2}}{1+\kappa_{2}}-\sigma_{2}, \quad \lambda_{03}=-\sigma_{Z}
$$


From (2.3), we see that this critical point is an unstable saddle.

\subsection{Prey only Critical Points A and C}

When $P_{1} \neq 0$, we obtain the prey only critical point $A$, given by $\left(P_{1}, P_{2}, Z, N\right)=$ $\left(P_{A}, 0,0, N_{A}\right)$, where

$$
P_{A}=1-\frac{\sigma_{1} \kappa_{1}}{\mu_{1}-\sigma_{1}}, \quad N_{A}=\frac{\sigma_{1} \kappa_{1}}{\mu_{1}-\sigma_{1}}, \quad N_{A}=1-P_{A}
$$

with stability given by

$$
\lambda_{A 1}=-\frac{\mu_{1} \kappa_{1} P_{A}}{\left(N_{A}+\kappa_{1}\right)^{2}}, \quad \lambda_{A 2}=\frac{\mu_{2} N_{A}}{N_{A}+\kappa_{2}}-\sigma_{2}, \quad \lambda_{A 3}=\frac{\phi_{1}\left(1-\psi_{1}\right) P_{A}}{1+\epsilon_{1} P_{A}}-\sigma_{Z} .
$$

$\lambda_{A 1}$ is always negative, while the remaining two eigenvalues can change sign.

When $P_{2} \neq 0$, we obtain the prey only critical point $C$, given by $\left(P_{1}, P_{2}, Z, N\right)=$ $\left(0, P_{C}, 0, N_{C}\right)$, where

$$
N_{C}=\frac{\sigma_{2} \kappa_{2}}{\mu_{2}-\sigma_{2}}, \quad P_{C}=1-N_{C}
$$

The eigenvalues are given by

$$
\lambda_{C 1}=-\frac{\mu_{2} \kappa_{2} P_{C}}{\left(N_{C}+\kappa_{2}\right)^{2}}, \quad \lambda_{C 2}=\frac{\mu_{1} N_{C}}{N_{C}+\kappa_{1}}-\sigma_{1}, \quad \lambda_{C 3}=\frac{\phi_{2}\left(1-\psi_{2}\right) P_{C}}{1+\epsilon_{2} P_{C}}-\sigma_{Z} .
$$

Again $\lambda_{C 1}<0$, while $\lambda_{C 2}$ and $\lambda_{C 3}$ can take either sign. 


\subsection{Dual Prey-only Critical Point B}

When $Z=0,(2.2)$ gives $f=0=g$, while $h \neq 0$, so that

$$
N_{B}=\frac{\sigma_{1} \kappa_{1}}{\mu_{1}-\sigma_{1}}=\frac{\sigma_{2} \kappa_{2}}{\mu_{2}-\sigma_{2}}=1-P_{1 B}-P_{2 B}
$$

Equation (3.6) can only be satisfied for certain parameter choices. However a linear stability analysis gives one of the eigenvalues as $\lambda_{1 B}=h$, but the resource sign condition for $Z$, evaluated along the line of maximum resource provides the parameter constraint that $h>0$. Therefore, the dual-prey critical point is always unstable.

\subsection{Predator-Prey Critical Point D}

When $P_{2}=0$, we obtain the $P_{1 D}, Z_{D}$ critical point. While the expressions for this critical point are independent of $\rho$, its stability is not. Moreover, we correct equation (A.20) of Cropp and Norbury [2009], which omitted a factor in the discriminant. Setting $f=0=h$ gives

$$
P_{1 D}=\frac{\sigma_{Z}}{\phi_{1}\left(1-\psi_{1}\right)-\epsilon_{1} \sigma_{Z}}, \quad Z_{D}=\left(\frac{1+\epsilon_{1} P_{1 D}}{\phi_{1}}\right)\left(\frac{\mu_{1} N_{D}}{N_{D}+\kappa_{1}}-\sigma_{1}\right)
$$

Since $Z_{D}=1-N_{D}-P_{1 D}$, we can eliminate $Z_{D}$ to obtain a quadratic equation for $N_{D}$ :

$$
d_{2} N_{D}^{2}+d_{1} N_{D}+d_{0}=0
$$


where

$$
\begin{aligned}
& d_{2}=\phi_{1}, \\
& d_{1}=\phi_{1}\left(P_{1 D}-1+\kappa_{1}\right)+\left(\mu_{1}-\sigma_{1}\right)\left(1+\epsilon_{1} P_{1 D}\right), \\
& d_{0}=-\sigma_{1} \kappa_{1}\left(1+\epsilon_{1} P_{1 D}\right)-\phi_{1} \kappa_{1}\left(1-P_{1 D}\right) .
\end{aligned}
$$

One eigenvalue for the stability of $D$ is

$$
\lambda_{1 D}=\frac{\mu_{2} N_{D}}{N_{D}+\kappa_{2}}-\frac{\phi_{2} Z_{D}}{1+\rho \epsilon_{1} P_{1 D}}-\sigma_{2}
$$

which depends upon $\rho$. The other two eigenvalues satisfy

$$
\lambda^{2}-P_{1 D} f_{D P_{1}} \lambda-P_{1 D} Z_{D} f_{D Z} h_{D P_{1}}=0,
$$

where $f_{D P_{1}}, f_{D Z}, h_{D P_{1}}$ are derivatives of $f$ and $h$ with respect to $P_{1}$ and $Z$, evaluated at the critical point $D$ :

$$
\begin{aligned}
f_{D P_{1}} & =-\frac{\mu_{1} \kappa_{1}}{\left(N_{D}+\kappa_{1}\right)^{2}}+\frac{\phi_{1} \epsilon_{1} Z_{D}}{\left(1+\epsilon_{1} P_{1 D}\right)^{2}}, \\
f_{D Z} & =-\frac{\mu_{1} \kappa_{1}}{\left(N_{D}+\kappa_{1}\right)^{2}}-\frac{\phi_{1}}{1+\epsilon_{1} P_{1 D}}, \\
h_{D P_{1}} & =\frac{\phi_{1}\left(1-\psi_{1}\right)}{\left(1+\epsilon_{1} P_{1 D}\right)^{2}} .
\end{aligned}
$$

\subsection{Predator-Prey Critical Point F}

When $P_{1}=0$, we obtain the $P_{2 F}, Z_{F}$ critical point. The expressions for $P_{2 F}, Z_{F}, N_{F}$ are readily obtained from those for critical point $D$ by simply replacing the subscript 
1 by the subscript 2 in the above. Thus

$$
P_{2 F}=\frac{\sigma_{Z}}{\phi_{2}\left(1-\psi_{2}\right)-\epsilon_{2} \sigma_{Z}}, \quad Z_{F}=\left(\frac{1+\epsilon_{2} P_{2 F}}{\phi_{2}}\right)\left(\frac{\mu_{2} N_{F}}{N_{F}+\kappa_{2}}-\sigma_{2}\right),
$$

and $Z_{F}=1-N_{F}-P_{2 F}$, where $N_{F}$ satisfies

$$
f_{2} N_{F}^{2}+f_{1} N_{F}+f_{0}=0
$$

where

$$
\begin{aligned}
& f_{2}=\phi_{2}, \\
& f_{1}=\phi_{2}\left(P_{2 F}-1+\kappa_{2}\right)+\left(\mu_{2}-\sigma_{2}\right)\left(1+\epsilon_{2} P_{2 F}\right), \\
& f_{0}=-\sigma_{2} \kappa_{2}\left(1+\epsilon_{2} P_{2 F}\right)-\phi_{2} \kappa_{2}\left(1-P_{2 F}\right) .
\end{aligned}
$$

The eigenvalues for the linear stability of $F$ are given by

$$
\lambda_{1 F}=\frac{\mu_{1} N_{F}}{N_{F}+\kappa_{1}}-\frac{\phi_{1} Z_{D}}{1+\rho \epsilon_{2} P_{2}}-\sigma_{1},
$$

and the roots of

$$
\lambda^{2}-P_{2 F} g_{F P_{2}} \lambda-P_{2 F} Z_{F} g_{F Z} h_{F P_{2}}=0,
$$


where again the coefficients $g_{F P_{2}}, g_{F Z}, h_{F P_{2}}$ are derivative of $g$ and $h$, evaluated at the critical point $F$ :

$$
\begin{aligned}
g_{F P_{2}} & =-\frac{\mu_{2} \kappa_{2}}{\left(N_{F}+\kappa_{2}\right)^{2}}+\frac{\phi_{2} \epsilon_{2} Z_{F}}{\left(1+\epsilon_{2} P_{2 F}\right)^{2}}, \\
g_{F Z} & =-\frac{\mu_{2} \kappa_{2}}{\left(N_{F}+\kappa_{2}\right)^{2}}-\frac{\phi_{2}}{1+\epsilon_{2} P_{2 F}}, \\
h_{F P_{2}} & =\frac{\phi_{2}\left(1-\psi_{2}\right)}{\left(1+\epsilon_{2} P_{2 F}\right)^{2}} .
\end{aligned}
$$

\subsection{Predator-Prey-Prey Critical Point E}

The final critical point is the interior critical point $E$ in which $\left(P_{1}, P_{2}, Z\right) \neq(0,0,0)$ and obtained by solving $f=g=h=0$. In Cropp and Norbury [2009] the discriminate limit of this critical point was calculated numerically by iteratively solving quadratic equations for $P_{1}$ and $Z$ in terms of $P_{2}$, and imposing the constraint that $N=1-$ $P_{1}-P_{2}-Z$ in order to determine the corresponding $N$. We adopted two approaches. One involved following Cropp and Norbury [2009] for the discriminate case, but in addition solving a quadratic equation for $N$ in terms of $P_{2}$ and using the constraints

$$
0<P_{1}, P_{2}, Z, N<1, \quad N+P_{1}+P_{2}+Z=1,
$$

to determine the appropriate values for the nutrient, the two prey and the predator. The second approach was to integrate equations (2.3) numerically, to produce bifurcation transition diagrams using both the maximum and minimum values of oscillatory solutions over each cycle. We chose to fix the value of $\rho$ and increase/decrease the predator mortality $\sigma_{Z}$. This enabled us to identify two Hopf bifurcation points in the two parameter $\left(\rho, \sigma_{Z}\right)$ plane. 
We note that the foraging transition parameter $\rho$ does not appear in expressions for the locations of any of the boundary equilibrium points (A - D, F) and hence only affects the location of the interior point E. However, it is involved in the stability properties of the two predator-prey boundary points $(\mathrm{D}, \mathrm{F})$ through the eigenvalue of the zero population at each point $\left(P_{2}\right.$ at $\mathrm{D}$ and $P_{1}$ at $\left.\mathrm{F}\right)$ in addition to the interior point E. The zooplankton mortality parameter $\sigma_{Z}$ does not appear in expressions for the locations or eigenvalues of the phytoplankton boundary equilibrium points (A C) but appears in all other points.

\subsection{Stability Curves}

Figure 1 summarises both the linear stability boundaries that we have found from stability analyses of the various critical points, and the computation of bifurcation transition curves, using the Table 1 parameter values for the transitionalist $N P P Z$ model. This is the first time that such boundaries have been calculated for this model. The discriminate case of $\rho=0$ is along the $\sigma_{Z}$ axis, while the indiscriminate case corresponds to the vertical section through $\rho=1$. Transitional values of $0<\rho<1$ result in a smooth transition between the two ecologically distinct foraging strategies, and increases of $\rho>1$ continue the smooth transition from chaos to stability as the foraging strategy changes and is enhanced. We note that while (3.10) and (3.16) suggest that increases in $\rho$ could potentially destabilise the boundary equilibrium points $(\mathrm{D}, \mathrm{F})$, the parameter set investigated does not produce critical values of $\rho$ in the range we have considered.

Figure 2 was produced by increasing $\sigma_{Z}$ from $\sigma_{Z}=0.19$ to $\sigma_{Z}=0.6$ and then decreasing $\sigma_{Z}$ to $\sigma_{Z}=0$ for $\rho=0$. For $0 \leq \sigma_{Z} \leq 0.11$, we find stable $\left(P_{2}, Z, N\right)$ 


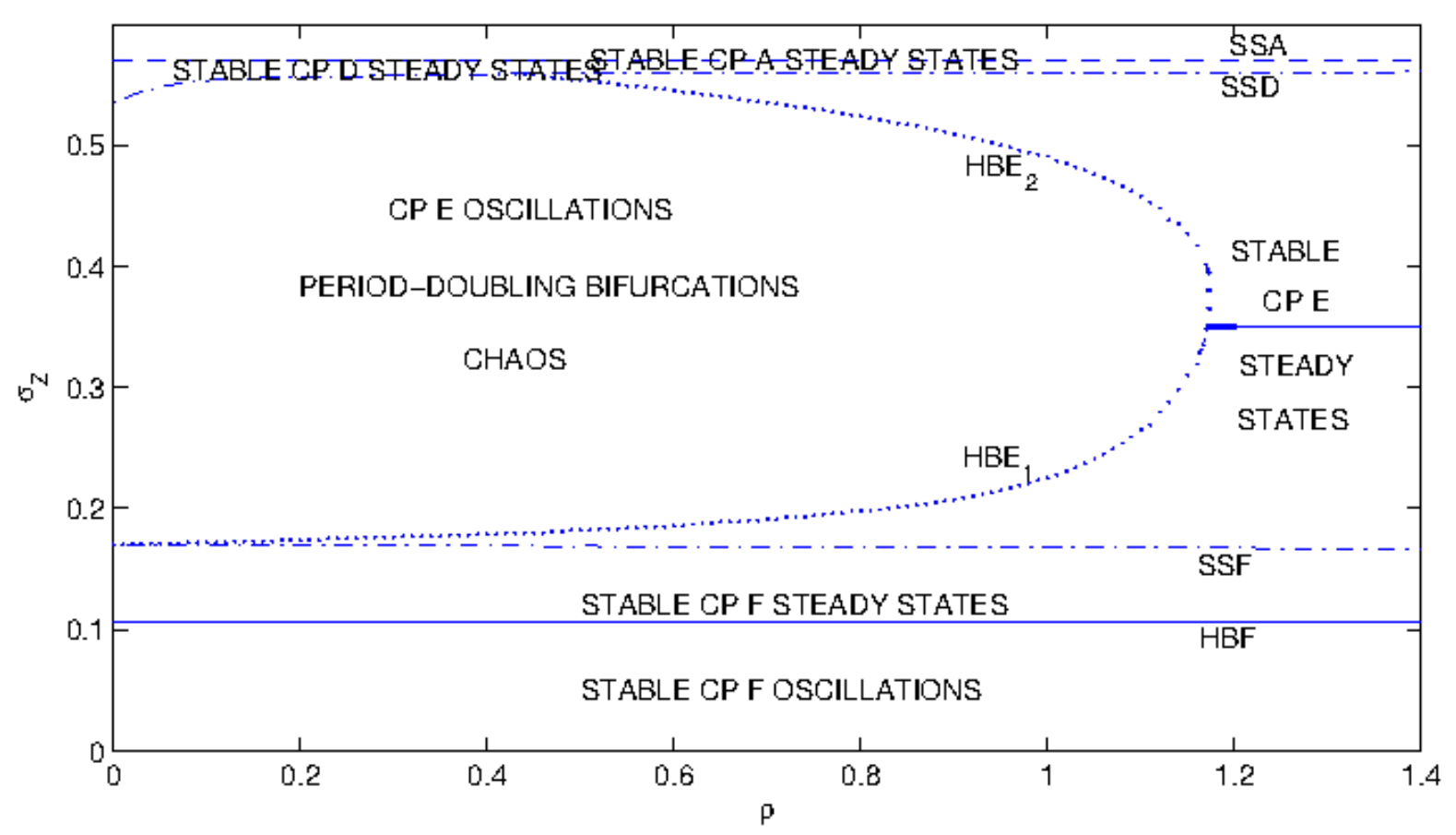

Figure 1: Linear stability boundaries and regions of stability for the various critical points, computed using the parameter values of Table 1, together with a summary of the nonlinear dynamics obtain via numerical integrations of the model and the calculation of bifurcation transition diagrams. Here $S S n$ denotes a transcritical steady state bifurcation for critical point $n$ and $H B n$ denotes a Hopf bifurcation for critical point $n$.

oscillations, which then lose stability to stable $F$ steady states. The latter persist until $\sigma_{Z} \approx 0.17$, where we find chaotic $\left(P_{1}, P_{2}, Z, N\right)$ solutions, with windows of periodicity (see Moroz et al. [2015]). These lose stability to $D$ steady states at $\sigma_{Z} \approx 0.533$ until $\sigma_{Z} \approx 0.57$ when $Z$ no longer is viable and stable steady $A$ states then persist.

In order to verify that we indeed have chaotic solutions, we calculated Lyapunov exponents for selected values of $\sigma_{Z}$ for $\rho=0$. To do this, we integrated (2.1), together with the associated 12 propagator equations, for either 10,000 and/or 15, 000 time 


\begin{tabular}{lll}
\hline$\sigma_{Z}$ & behaviour & Leading Lyapunov exponent \\
\hline 0.2 & chaotic & 0.0159 \\
0.3 & chaotic & 0.0276 \\
0.4 & chaotic & 0.0555 \\
\hline
\end{tabular}

Table 2: Lyapunov exponents for various $\sigma_{Z}$ when $\rho=0$

units, once transients had been removed, with a time step of 0.01 . Table 2 shows the leading Lypaunov exponents. We validated our code by repeating the exercise for the Lorenz equations with their classic parameter values of $(r, \sigma, b)=(28,10,8 / 3)$ and reproduced the value for the Lyapunov exponents of $0.906,0.0$ and -14.573 .

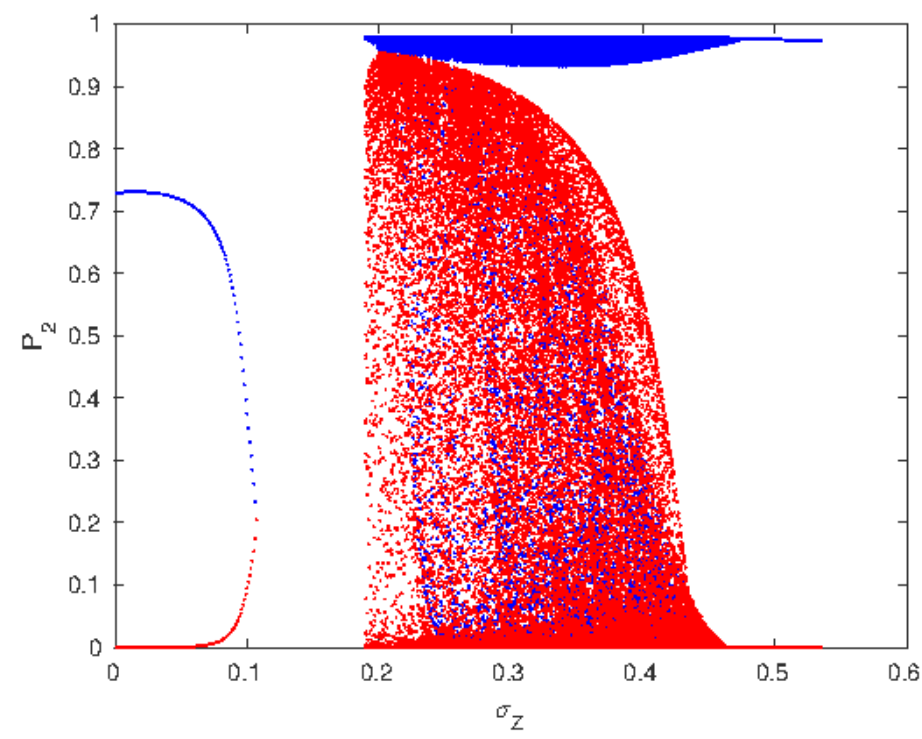

Figure 2: The bifurcation transition diagram for $\rho=0$. When $\sigma_{Z}$ is increased or decreased from $\sigma_{Z}=0.19$, we obtain the full diagram. When $\sigma_{Z}$ is increased from $\sigma_{Z}=0$, we only obtain the periodic states in $0 \leq \sigma_{Z} \leq 0.11$.

A very different and much simpler picture occurs when we increase $\sigma_{Z}$ from $\sigma_{Z}=0$. Stable $\left(P_{2}, Z, N\right)$ oscillations again occur for $0<\sigma_{Z}<0.11$, while $Z \rightarrow 0$ at 
$\sigma_{Z} \approx 0.18$, leaving a stable $C$ steady state.

The scenario is much simpler when $\rho=1$ (see figure 3 ). The behaviour follows

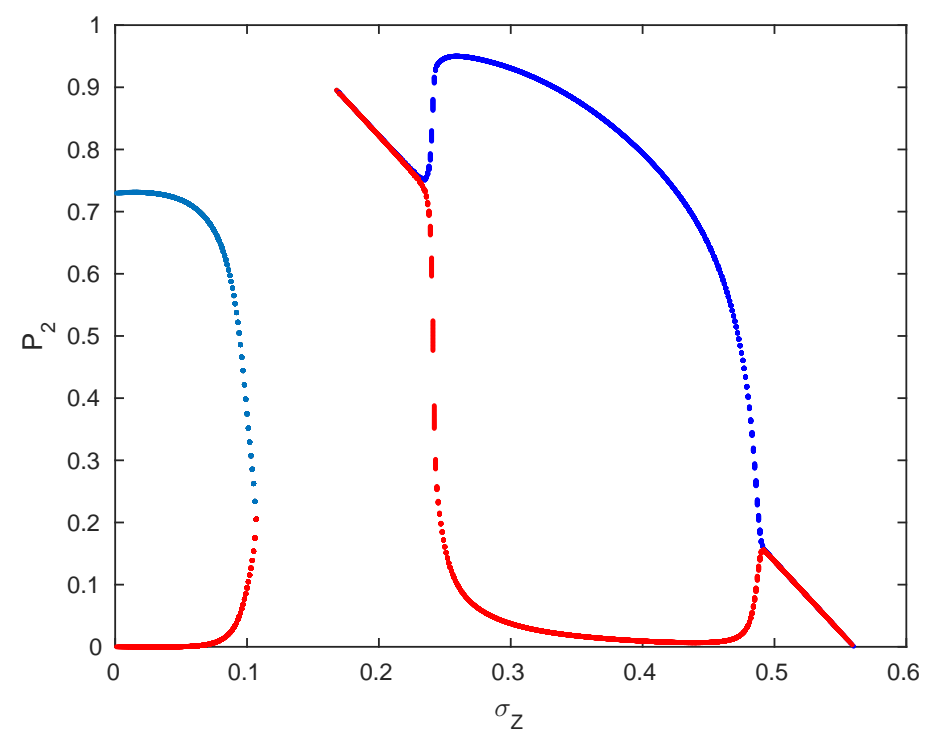

Figure 3: The bifurcation transition diagram for $\rho=1$. When $\sigma_{Z}$ is increased or decreased from $\sigma_{Z}=0.2$, we obtain the full diagram. When $\sigma_{Z}$ is increased from $\sigma_{Z}=0$, we only obtain the periodic states in $0 \leq \sigma_{Z} \leq 0.11$.

that for the $\rho=0$ case until $\sigma_{Z} \approx 0.17$, when we find stable steady $E$ states. These persist until $\sigma_{Z} \approx 0.225$ when stable simply periodic $\left(P_{1}, P_{2}, Z, N\right)$ solutions appear through a supercritical Hopf bifurcation $\left(H B E_{1}\right.$ in figure 1$)$, only to disappear in a second supercritical Hopf bifurcation $\left(H B E_{2}\right.$ in figure 1$)$ at $\sigma_{Z} \approx 0.491$. We then find stable steady $E$ states for $0.491<\sigma_{Z}<0.56$, which lose stability to stable $D$ steady states in $0.56<\sigma_{Z}<0.57$, before the final state of stable steady $A$ states for $\sigma_{Z}>0.57$. When $\sigma_{Z}$ is increased from $\sigma_{Z}=0$, we only obtain the periodic states in $0 \leq \sigma_{Z} \leq 0.11$. 


\section{Seasonal Forcing}

Phytoplankton blooms are population explosions in the presence of sunlight and nutrients, lasting from a few days to several weeks, until the nutrient has been consumed or they are grazed down by zooplankton. In temperate and polar oceans, phytoplankton blooms are closely associated with seasonal changes. Increasing solar irradiance and an associated shoaling of the mixed layer depth trap phytoplankton in a high-light, high-nutrient environment that provides ideal conditions for phytoplankton growth producing the classical "vernal bloom" described by Sverdrup [1953]. The increased availability of phytoplankton initiates a response in the zooplankton population. We now investigate the effects of seasonal forcing on the two special limits of the transitionalist model, namely the discriminate and indiscriminate cases. We introduce the forcing to the $\mu_{j}$ parameters $(j=1,2)$, which represent the maximum uptake of the nutrient $N$ by each phytoplankton population (see Table 1 ). In the forced scenario, the $\mu_{j}$ represent the average of the maximum rate of uptake of nutrient that is possible at different times of year:

$$
\mu_{j}(t)=(1+A \sin (\omega t)) \mu_{j}
$$

where $0 \leq A \leq 1$ is the amplitude of the forcing. The forcing frequency $\omega$ was chosen in two ways, one $\omega_{P}=2 \pi / 365$, based upon the phytoplankton time scale, and the second $\omega_{Z}=\omega_{P} / 9$, based upon the zooplankton time scale (so that 10, 000 time units correspond to $\approx 3$ years). 

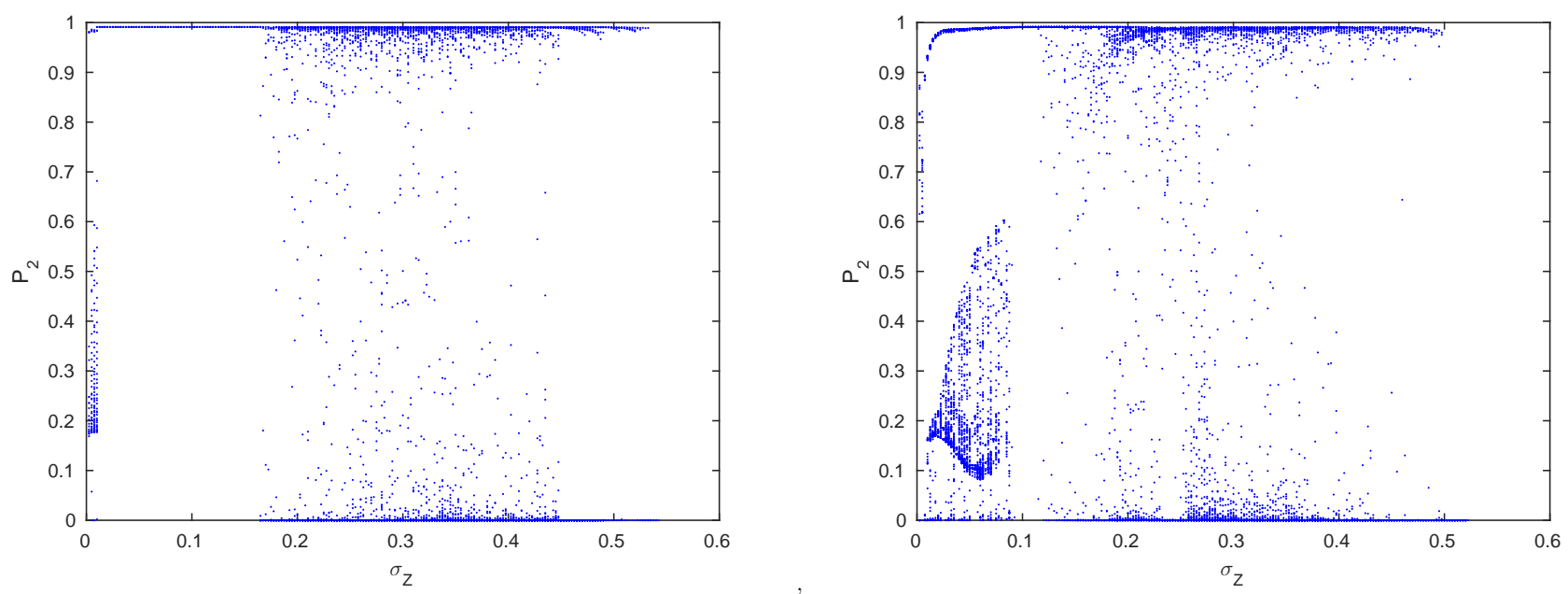

Figure 4: The plot of maximum values of the amplitude of $P_{2}$ over each cycle as $\sigma_{Z}$ varies for the discriminate case $\rho=0$ with a forcing amplitude of $A=1$. The left hand panel is for $\omega_{Z}$ and the right hand panel is for $\omega_{P}$.

\subsection{Discriminate case $\rho=0$}

When $\rho=0$ and with a forcing amplitude of $A=1$, we obtain chaotic solutions within the same range of values as for the case of no seasonal forcing. Figure 4 shows the bifurcation transition diagram for both the $\omega_{P}$ and $\omega_{Z}$ timescales. To produce these plot we followed the same approach as in the no forcing case. $\sigma_{Z}$ was increased from $\sigma_{Z}=0.19$ to $\sigma_{Z}=0.6$ and then decreased from $\sigma_{Z}=0.19$ to $\sigma_{Z}=0$. If instead $\sigma_{Z}$ is increased from $\sigma_{Z}=0.001$, we obtain figure 5 . In contrast with the case of no forcing, the main difference in the $\omega_{Z}$ case is for $\sigma_{Z}<0.166$, where we no longer find either steady $F$ states or periodic $\left(P_{2}, Z, N\right)$ solutions. Instead both $P_{1}$ and $Z$ go to zero, leaving steady $C$ states until $\sigma_{Z} \approx 0.003$ when $Z$ states re-emerge. With $\omega_{P}, Z$ is maintained throughout. 

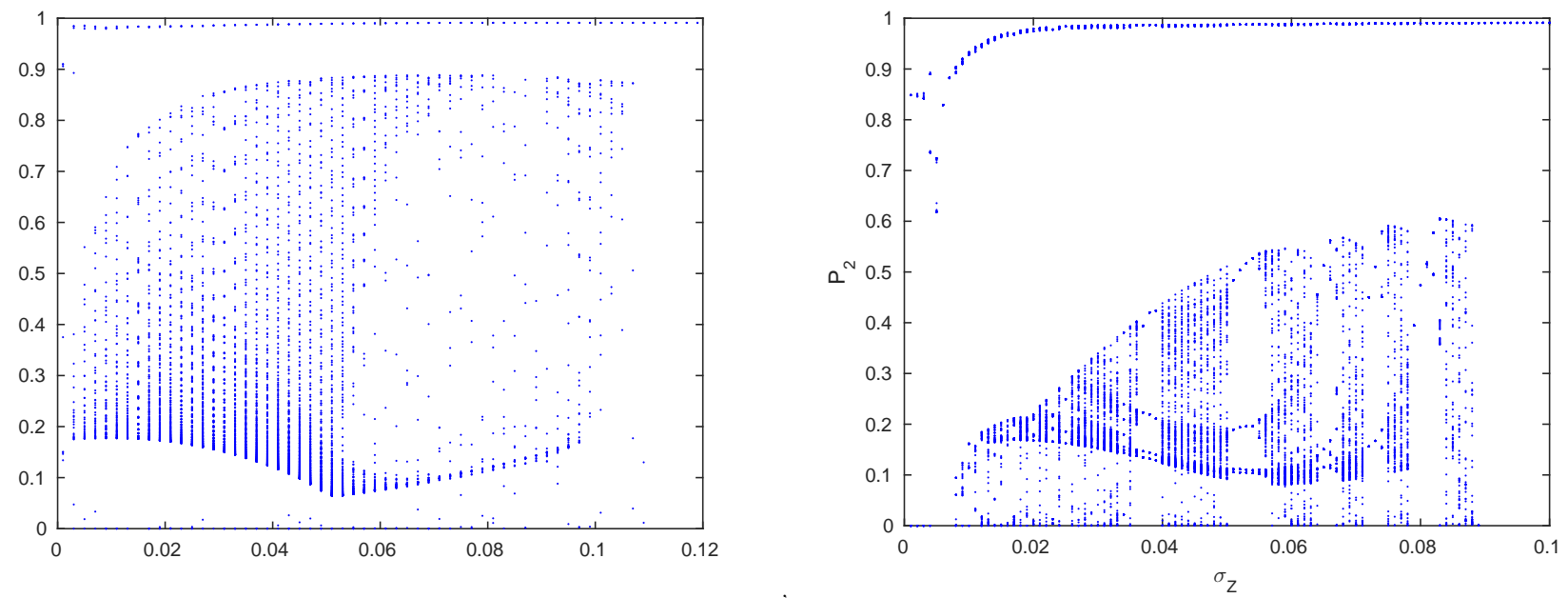

Figure 5: The plot of maximum values of the amplitude of $P_{2}$ over each cycle as $\sigma_{Z}$ is increased from $\sigma_{Z}=0.001$ for the discriminate case $\rho=0$ with a forcing amplitude of $A=1$ for $\omega_{Z}$ (left hand panel) and $\omega_{P}$ (right hand panel).

\subsection{Indiscriminate case $\rho=1$}

Seasonal forcing has a far bigger impact on the indiscriminate case of $\rho=1$ than on the discriminate case of $\rho=0$. Instead of simple periodic solutions, we find both chaotic and periodic states. Figure 6 shows the bifurcation transition diagram for $A=1$ with $\omega_{P}$.

Figure 7 shows time series of $P_{1}, P_{2}$ and $Z$, as well as a $3-\mathrm{D}$ phase portrait in the $\left(P_{1}, P_{2}, Z\right)$ plane for $\sigma_{Z}=0.3$. The periodic solutions in the unforced case have been replaced by a chaotic state in the forced state. Figure 8 shows a period-23 cycle for $\sigma_{Z}=0.51$ and $A=1$, produced by integrating (2.1) for 100,000 time units with a non-dimensional time step of 0.01 . 

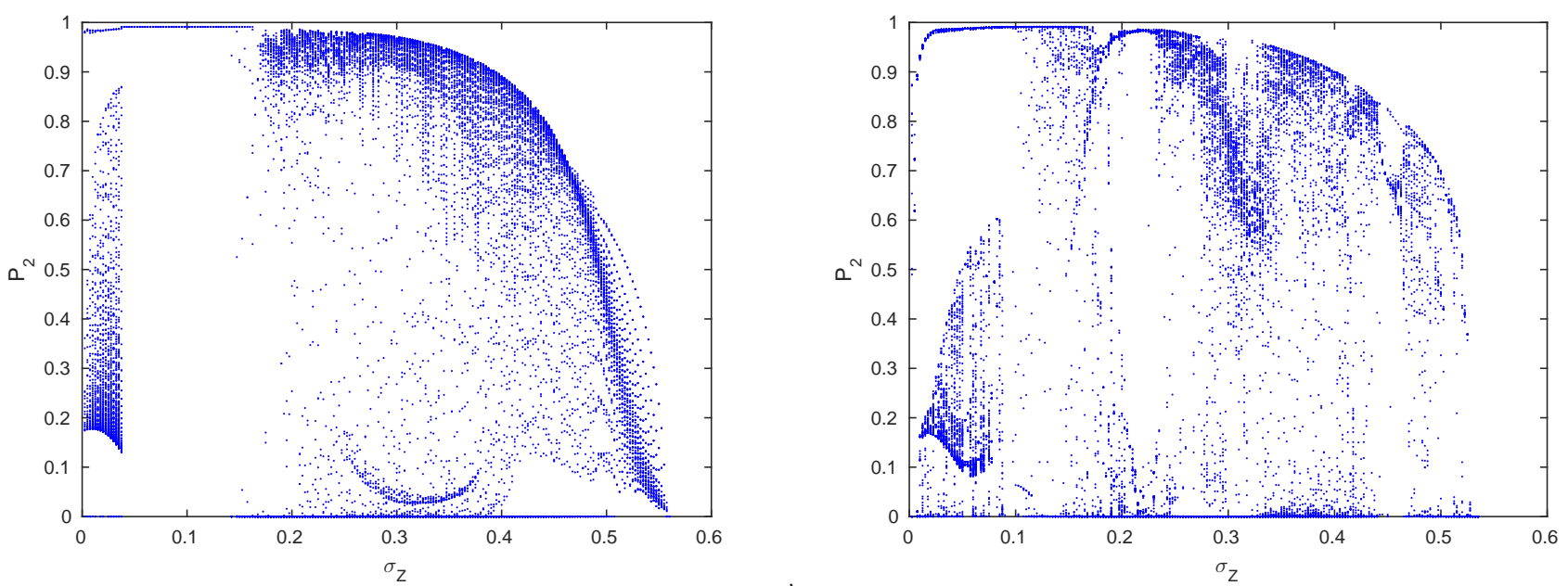

Figure 6: The plot of maximum values of the amplitude of $P_{2}$ over each cycle as $\sigma_{Z}$ varies for the indiscriminate case $\rho=1$ with a forcing amplitude of $A=1$ for $\omega_{Z}$ (left hand panel) and $\omega_{P}$ (right hand panel).
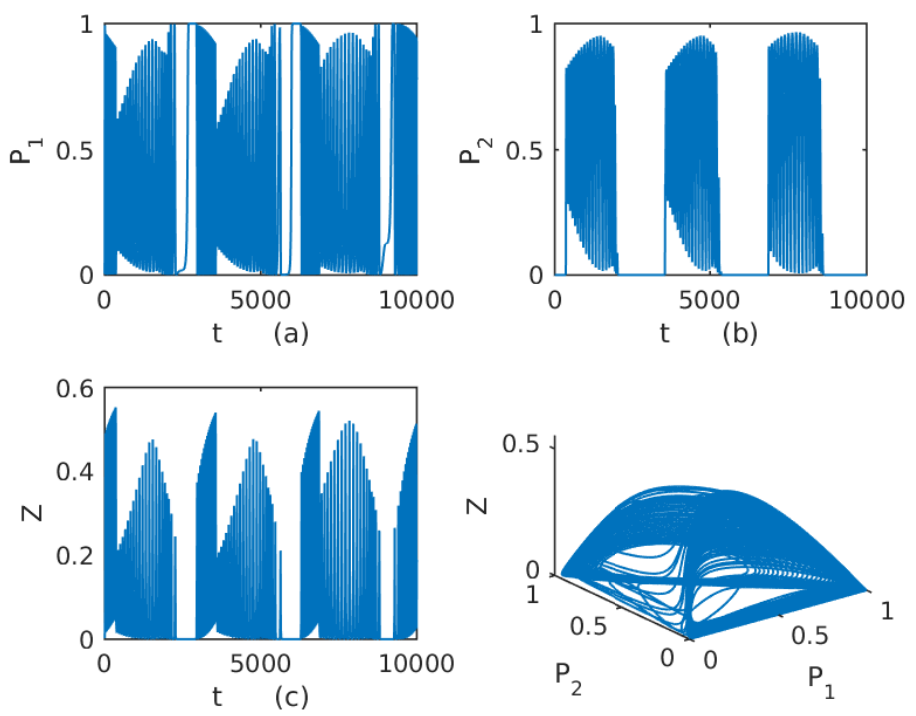

Figure 7: Time series of (a) $P_{1}$, (b) $P_{2}$, (c) $Z$ and (d) the 3 -D phase portrait $\left(P_{1}, P_{2}, Z\right.$ ) for $\sigma_{Z}=0.3$ for the indiscriminate case with a forcing amplitude of $A=1$ and $\omega_{Z}$. 


\subsection{Varying the Seasonal forcing amplitude}

It is interesting to investigate the effects of varying the forcing amplitude $A$ on the unforced dynamics. We chose to do this for both the discriminate and indiscriminate models for $\sigma_{Z}=0.3$, which gives one of the largest values for $P_{2}$ in each oscillatory cycle. For the discriminate case chaotic behaviour observed for $A=0$ is maintained over the entire region $0 \leq A \leq 1$. Figure 9 shows the indiscriminate case. The range of values for $P_{2 \max }$ gradually increases as $A$ increases, until they expand to include the whole range of values when $A>0.6$.

Figure 10 shows a quasiperiodic solution for $\sigma_{Z}=0.3$ and $A=0.5$.

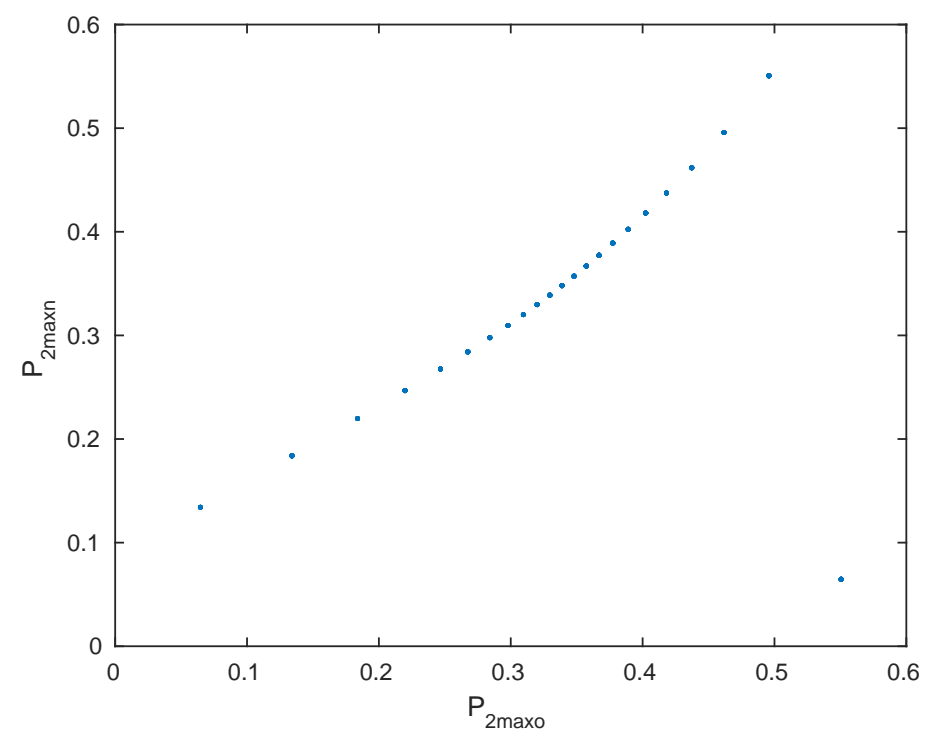

Figure 8: The plot of successive maxima of the amplitude of $P_{2}$ over each cycle for $\sigma_{Z}=0.51$ for the indiscriminate case with a forcing amplitude of $A=1$ and $\omega_{Z}$. 


\section{$5 \quad$ Different Functional Forms}

Bates et al. [2015] investigated a twenty-one dimensional system of coupled nonlinear odes to model a near-shore Antarctic food web using the Conservative-Normal approach. Their model used Holling Type III grazing functional forms and quadratic mortality terms which ensured the coexistence of all ecosystem members, as all boundary equilibrium points had at least one unstable eigenvalue. Numerical integrations of this model produced only stable interior steady states. This raises the interesting possibility that the functional forms used in Bates et al. [2015] have precluded the occurrence of chaotic dynamics in a high-dimensional, highly nonlinear model.

So far our studies have focused upon Holling Type II functional forms and linear mortality terms. We now investigate the consequences of moving towards Holling Type III functional forms and quadratic mortality and introduce these terms into our

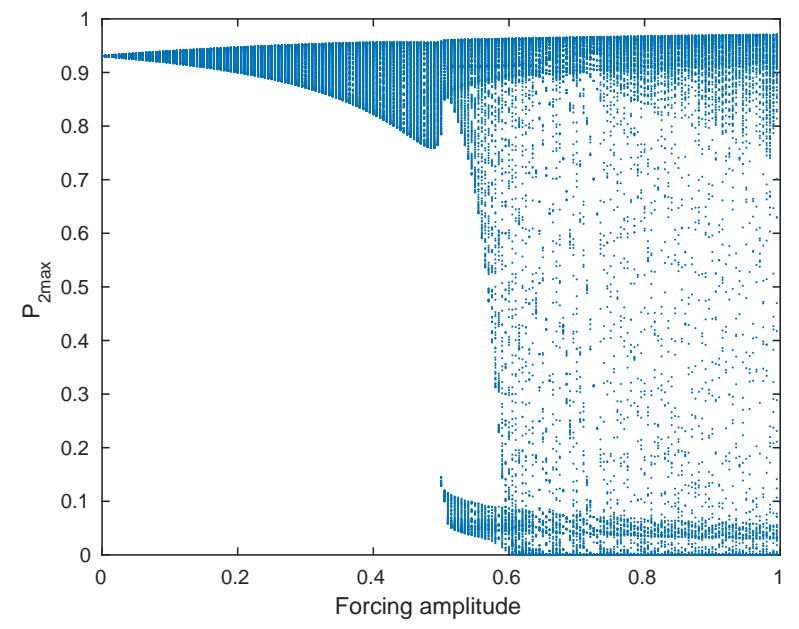

Figure 9: The plots of maximum values of the amplitude of $P_{2}$ over each cycle for $\sigma_{Z}=0.3$ for the indiscriminate case as a function of the forcing amplitude of $A=1$. 

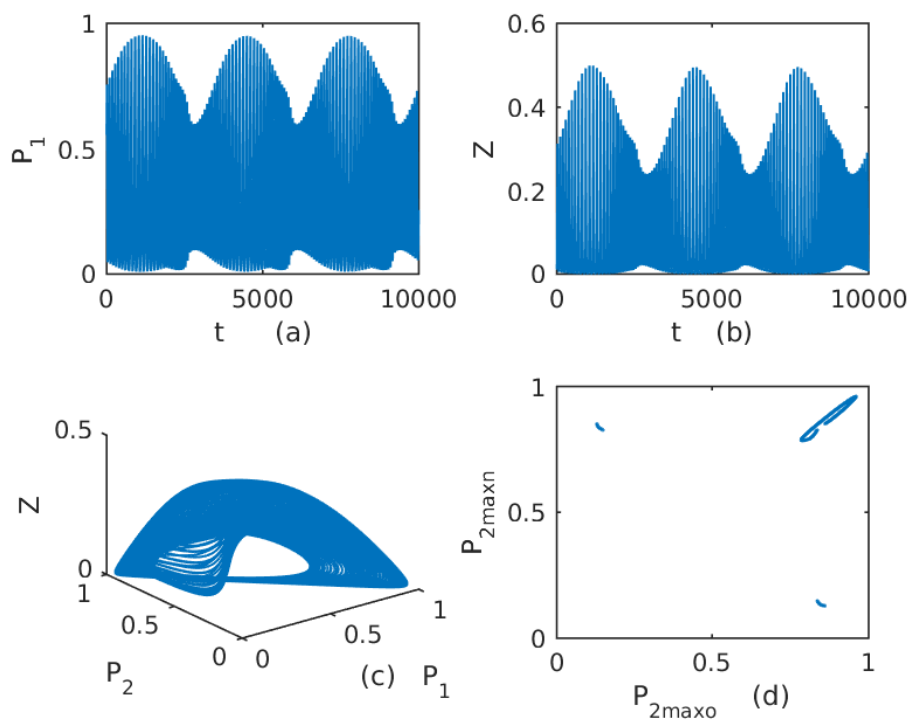

Figure 10: The plots of (a) $P_{1}(t)$, (b) $Z(t),\left(\right.$ c) the phase portrait in the $\left(P_{1}, P_{2}\right)$ plane and $(\mathrm{d})$ successive maximum values of the amplitude of $P_{2}$ over each cycle for $\sigma_{Z}=0.3$ for the indiscriminate case for $A=0.5$.

simple model (2.1). We consider the transitionalist system:

$$
\begin{aligned}
\dot{P}_{1} & =P_{1}\left[\frac{\mu_{1} N}{N+\kappa_{1}}-\frac{\phi_{1} Z P_{1}^{a}}{1+\epsilon_{1} P_{1} P_{1}^{a}+\rho \epsilon_{2} P_{2} P_{2}^{a}}-\sigma_{1} P_{1}^{a}\right]=P_{1} F\left(P_{1}, P_{2}, Z\right),( \\
\dot{P}_{2} & =P_{2}\left[\frac{\mu_{2} N}{N+\kappa_{2}}-\frac{\phi_{2} Z P_{2}^{a}}{1+\rho \epsilon_{1} P_{1} P_{1}^{a}+\epsilon_{2} P_{2} P_{2}^{a}}-\sigma_{2} P_{2}^{a}\right]=P_{2} G\left(P_{1}, P_{2}, Z\right),( \\
\dot{Z} & =Z\left[\frac{\phi_{1}\left(1-\psi_{1}\right) P_{1} P_{1}^{a}}{1+\epsilon_{1} P_{1} P_{1}^{a}+\rho \epsilon_{2} P_{2} P_{2}^{a}}+\frac{\phi_{2}\left(1-\psi_{2}\right) P_{2} P_{2}{ }^{a}}{1+\rho \epsilon_{1} P_{1} P_{1}^{a}+\epsilon_{2} P_{2} P_{2}^{a}}-\sigma_{Z} Z^{a}\right] \\
& =Z H\left(P_{1}, P_{2}, Z\right),
\end{aligned}
$$

together with the nutrient equation (2.2). When $a=0$ we obtain the Holling Type II system (2.1), while $a=1$ gives the fully quadratic equivalent of the Bates et al. [2015] system.

Because (5.1) is written in Kolmogorov form, the critical points from $\dot{P}_{1}=\dot{P}_{2}=$ 
$\dot{Z}=0$ give:

$$
P_{1}=0 \text { or } F=0, \quad P_{2}=0 \text { or } G=0, \quad Z=0 \text { or } H=0,
$$

with linear stability given by the Jacobian matrix:

$$
J=\left(\begin{array}{ccc}
F+P_{1} F_{P_{1}} & P_{1} F_{P_{2}} & P_{1} F_{Z} \\
P_{2} G_{P_{1}} & G+P_{2} G_{P_{2}} & P_{2} G_{Z} \\
Z H_{P_{1}} & Z H_{P_{2}} & H+Z H_{Z}
\end{array}\right)
$$

evaluated at each critical point. Despite the presence of the power $a$, using the results of $\S 3$, it is straightforward to check that all of the boundary points (critical points for which one or more of the populations are zero) are unstable.

For example, consider the critical point $P_{1}=0$, but $P_{2} \neq 0, Z \neq 0$. The Jacobian matrix (5.3) reduces to

$$
J=\left(\begin{array}{ccc}
F & 0 & 0 \\
P_{2} G_{P_{1}} & P_{2} G_{P_{2}} & P_{2} G_{Z} \\
Z H_{P_{1}} & Z H_{P_{2}} & Z H_{Z}
\end{array}\right)
$$

One eigenvalue is given by

$$
\lambda=F\left(0, P_{2}, Z\right)=\frac{\mu_{1} N}{N+\kappa_{1}}>0
$$

and so this critical point is unstable. This leaves only the interior predator-prey-prey point. Because of the algebraic complexity of determining this critical point explicitly, 
instead we decided to determine how the chaotic dynamics for the discriminate case of $a=0$ and $\rho=0$ was affected by increasing $a$ from $a=0$ to $a=1$. We again chose $\sigma_{Z}=0.3$ as a typical value in the chaotic regime. When $a=0$ we have chaotic states. However when $a=0.02$ we obtained a simple periodic solution, and stable interior steady states for $a \geq 0.04$. For the indiscriminate case, $a=0$ gives a simple periodic solution, while for $a \geq 0.01$, we obtained stable interior steady states. This also includes the Holling Type III limit of $a=1$. We are therefore able to reproduce the results of Bates et al [2015] for a much lower dimensional ecosystem. While these are isolated numerical experiments, it seems that the chaotic dynamics is sensitive to the right choice of functional form, and somewhat counter-intuitively, that chaos might be suppressed in systems that are "too nonlinear".

Although the unforced system (5.1) only supports stable interior steady states, the inclusion of seasonal forcing produces periodic behaviour. Figure 11 shows plots of the maxima and minima of $P_{2}$ as $\sigma_{Z}$ is increased with a forcing amplitude $A=1$ for (5.1) with $a=1$ for both (a) $\omega_{P}$ and (b) $\omega_{Z}$.

\section{Discussion}

In this paper we examined the dynamical properties of a simple plankton ecosystem model to provide some insights into the role of various processes and choices made by modellers in determining the predictions produced by such models. This is an interesting and important topic, as relatively complex plankton models are being developed for inclusion in climate models to represent important biogeochemical cycling processes in the global oceans. Plankton ecosystem models also underpin most 

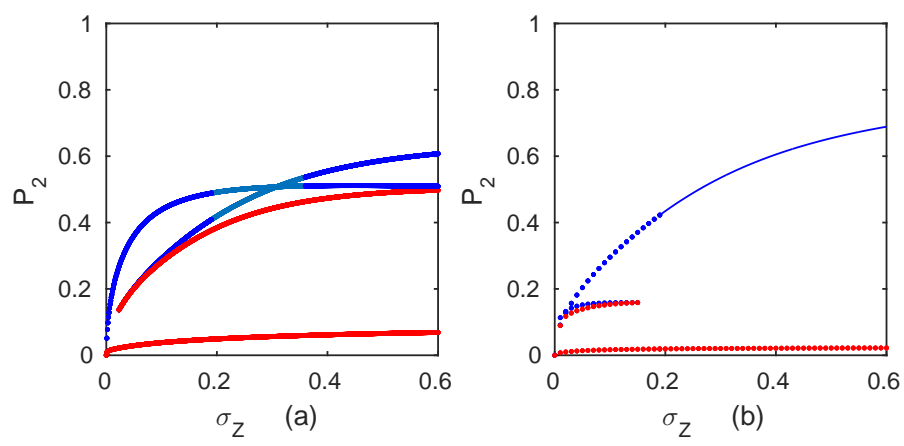

Figure 11: The plots of maxima (blue) and minima (red) of $P_{2}$ as $\sigma_{Z}$ is varied for the indiscriminate case of (5.1) with $a=1$ and $A=1$ for (a) $\omega_{P}$, (b) $\omega_{Z}$.

contemporary fisheries management models, and in both these applications, the models are required to predict the dynamics of these important systems in response to substantial external perturbations.

Our first results suggest that the choice of foraging function is an important influence on system dynamics, with a discriminate grazer being more likely to induce chaotic dynamics into a system than an indiscriminate grazer. The smooth transition between these grazing behaviours, that are ecologically ambiguous as only the extreme cases are usually considered, has not been investigated previously but supports the robustness of our results. It is interesting that most complex phytoplankton models use indiscriminate grazing terms, perhaps according to rubrics developed from numerical solutions that certain terms are "stabilising" (for example, quadratic mortality is often considered a "stabilising" term by numerical modellers). Whether plankton 
systems are naturally stable or chaotic is a moot point, with some authors such as Huisman and Weissing [1999] arguing that chaos is necessary to explain the diversity observed in plankton populations in the global oceans. The use of indiscriminate grazing terms in complex plankton models may be useful in the short term to provide more computationally tractable models. However recent work, showing that omnivorous planktonic grazers may change their feeding behaviour in response to changes in temperature (Wilken et al. [2013]) suggests that this issue requires a lot more attention for future models that may be more focussed on determining the impacts of climate change on marine plankton than the current models that focus on the impact of plankton on climate.

The application of seasonal forcing to the model generated chaotic dynamics irrespective of whether the unforced model was chaotic or not. However, some attributes of the underlying stability diagram (Fig 1) remain evident in the forced chaotic dynamics, with chaos generally being confined to the region where the zooplankton is obligate on $P_{1}$. Some chaotic dynamics are, however, evident for both discriminate and indiscriminate grazing cases when the zooplankton is not obligate on $P_{1}$, but can survive on either $P_{1}$ or $P_{2}\left(0<\sigma_{Z}<0.17\right)$. Increasing the amplitude of the seasonal forcing (small amplitude forcings are conceptually equivalent to temperate regions, large amplitudes to polar regions) had little effect on the discriminate grazing model, as it maintained its large amplitude oscillations. However, the indiscriminate grazing model, which had stable or simple limit cycle dynamics in the unforced scenario, evidenced increasing amplitude chaotic dynamics as the amplitude of the seasonal forcing increased. Results were similar whether a phytoplankton-based or zooplankton-based forcing period was used. 
Our final major result has useful implications for applications of plankton models in climate change and fisheries management as it suggests that chaotic dynamics may be "tamed" by the use of specific functional forms, and brings the potential to design even very complicated models to have specific properties into the realm of possibility. Somewhat surprisingly, chaotic dynamics in these models appears to be able to be eliminated by making the model more nonlinear, specifically by replacing linear mortality with quadratic mortality, and by replacing hyperbolic Holling Type II grazing terms with sigmoidal Holling Type III terms. Recent work exemplified in the application of Bates et al. [2015] provided clear heuristics describing how these terms may be used to ensure coexistence in complex plankton models. The results here suggest that the influence of these terms may extend to determining whether a model can have chaotic dynamics or not. These results provide a conjecture of interest to numerical modellers of marine plankton systems when designing new models: the use of Holling Type III grazing in conjunction with quadratic mortality for all population loss terms provides a model where all populations coexist in stable equilibrium. Conversely, chaotic dynamics in an existing model may be quashed by changing grazing from discriminate to indiscriminate or from Holling Type II to Holling Type III, and mortality from linear to quadratic.

The most interesting result in more general terms however remains the tantalising suggestion of these results that models may be to "too nonlinear" for chaos, that in fact the general rubric that chaos requires, or is more commonly found associated with, nonlinearity is a simplification. Nonlinearity, like many things in life, has to be "just right". 


\section{References}

M.L.Bates, S.M. Bengtson Nash, D.W. Hawker, J. Norbury, J.S. Stark, R.A. Cropp. Construction of a trophically complex near-shore Antarctic food web model using the Conservative-Normal framework with structural coexistence. J. Mar. Syst. 145, 1-14. 2015.

P. Bork, C. Bowler, C. de Vargas, G. Gorsky, E. Karsenti and P. Wincker. Tara Oceans studies plankton at planetary scale. Science, 348, 873, 2015.

R.J. Charlson, J.E. Lovelock, M.O. Andreae and S.G. Warren. Oceanic phytoplankton, atmospheric sulphur, cloud albedo and climate. Nature, 326, 655-661, 1987.

R. A. Cropp and J. Norbury. Parameterizing plankton functional type models: insights from a dynamical systems perspective. Journal of Plankton Research, 31, 939-963, 2009.

R. A. Cropp and J. Norbury. Constructing Ecologies. J. Theor. Biology, 294, 1-8, 2012a.

R. A. Cropp and J. Norbury. The mechanisms of coexistence and competitive exclusion in complex plankton ecosystem models. Ecosystems, 15, 200-212, 2012b.

R. A. Cropp, I.M. Moroz and J.Norbury. The role of grazer predation choices in the dynamics of consumer-resource based ecologies. Submitted, 2015.

P. Falkowski. The power of plankton. Nature, 483, 817-820, 2012.

K.J. Flynn, D.K. Stoecker, A. Mitra, J.A. Raven, P.M. Glibert, P.J. Hansen, E. Graneli and J.M. Burkholder. Misuse of the phytoplankton-zooplankton di- 
chotomy: the need to assign organisms as mixotrophs within plankton functional types. Journal of Plankton Research, 35:3-11. 2013.

J. Huisman and F.J. Weissing. Biodiversity Of Plankton By Species Oscillations And Chaos. Nature, 402:407-410. 1999.

L. Kwiatkowski, A. Yool, J.I. Allen, T.R. Anderson, R. Barciela, E.T. Buitenhuis, M. Butenschn, C. Enright, P.R. Halloran, C. Le Quéré, L. de Mora, M.F. Racault, B. Sinha, I.J. Totterdell and P.M. Cox. iMarNet: an ocean biogeochemistry model intercomparison project within a common physical ocean modelling framework. Biogeosciences, 11, 7291-7304, 2014.

C. Le Quéré, S.P. Harrison, I.C. Prentice, E.T. Buitenhuis, O. Aumont, L. Bopp, H. Claustre, L.C. Da Cunha, R. Geider, X. Giraud, C. Klaas, K.E. Kohfeld, L. Legendre, M. Manizza, T. Platt, R.B. Rivkin, S. Sathyendranath, J. Uitz, A.J. Watson and D. Wolf-Gladrow. Ecosystem dynamics based on plankton functional types for global ocean biogeochemistry models. Global Change Biology, 11, 2016-2040, 2005.

A. Mitra, K.J. Flynn, J.M. Burkholder, T. Berge, A. Calbet, J.A. Raven, E. Graneli, P.M. Glibert, P.J. Hansen, D.K. Stoecker, F. Thingstad, U. Tillmann, S. Vage, S. Wilken and M.V. Zubkov. The role of mixotrophic protists in the biological carbon pump. Biogeosciences, 11:995-1005. 2014.

I.M. Moroz, R. A. Cropp and J.Norbury. A simple plankton model with complex behaviour. Submitted, 2015.

H.V. Sverdrup. On conditions for the vernal blooming of phytoplankton. Journal Conseil International Exploration du Mer, 18:287-295. 1953. 
S. Wilken, J. Huisman, S. Naus-Wiezer and E. Van Donk. Mixotrophic organisms become more heterotrophic with rising temperature. Ecology Letters, 16:225-233. 2013. 